Théologiques

Théologiques

\title{
Le débat de la pertinence existentielle de la théodicée. Positions, enjeux et éclairages sur les rapports entre théorie et pratique
}

\section{Lamphone Phonevilay}

Volume 15, numéro 1, 2007

Mémoires et usages religieux de l'espace

URI : https://id.erudit.org/iderudit/017645ar

DOI : https://doi.org/10.7202/017645ar

Aller au sommaire du numéro

Éditeur(s)

Faculté de théologie et de sciences des religions, Université de Montréal

ISSN

1188-7109 (imprimé)

1492-1413 (numérique)

Découvrir la revue

Citer cet article

Phonevilay, L. (2007). Le débat de la pertinence existentielle de la théodicée. Positions, enjeux et éclairages sur les rapports entre théorie et pratique. Théologiques, 15(1), 153-170. https://doi.org/10.7202/017645ar

\section{Résumé de l'article}

Dans les années 1980, deux auteurs, Kenneth Surin et Terrence W. Tilley, ont amorcé un débat quant à la pertinence existentielle de la théodicée, en critiquant fortement l'entreprise de cette dernière. Leurs positions ont provoqué de vives réactions dans l'arène universitaire, celles-ci s'avérant soit favorables, soit défavorables à la théodicée. L'article expose les positions des principaux protagonistes du débat et propose quelques réflexions sur le thème des rapports entre théorie et pratique qui s’y dégage. À l'horizon de ce débat se dessine une vision binaire des choses, que l'auteur critique pour dénouer les impasses actuelles dans lesquelles celui-ci semble être plongé.
Tous droits réservés @ Faculté de théologie et de sciences des religions, Université de Montréal, 2007
Ce document est protégé par la loi sur le droit d'auteur. L'utilisation des services d'Érudit (y compris la reproduction) est assujettie à sa politique d'utilisation que vous pouvez consulter en ligne.

https://apropos.erudit.org/fr/usagers/politique-dutilisation/ 


\section{Le débat de la pertinence existentielle de la théodicée. Positions, enjeux et éclairages sur les rapports entre théorie et pratique}

Lamphone PHONEVILAY

Étudiant au doctorat en cotutelle Université de Montréal (sciences des religions) École des Hautes Études en Sciences Sociales de Paris (sociologie)

La question du mal s'avère une question fondamentale pour tout croyant, qu'il doit éventuellement se poser un jour ou l'autre. Cette question, et la réponse qui s'ensuit, prend souvent la forme d'une théodicée qui tente d'articuler, en régime monothéiste, mais plus particulièrement chrétien, l'idée de l'existence d'un Dieu tout-puissant et infiniment bon. De manière plus schématique, cette question se formule de la manière suivante: "Si Dieu est tout-puissant et infiniment bon, pourquoi permet-il l'existence du mal dans le monde? " À cette question, les théologies essaient d'apporter leurs propres éléments de réponse. Certaines revendiquent une préséance totale de la liberté humaine, dont le corollaire inévitable serait la possibilité de faire le mal chez l'être humain. D'autres prescrivent une pédagogie divine, où le mal et les épreuves qui en découlent pour les êtres humains constituent autant d'occasions pour ces derniers de s'améliorer et de se dépasser spirituellement. D'autres encore font appel à l'œuvre du démon, qui serait à l'origine du mal par les tentations qu'il offre aux humains ${ }^{1}$.

Le nombre impressionnant de théodicées qui ont été élaborées en contexte chrétien témoigne de la complexité de la question du mal. Celleci peut prendre différentes formes et peut être typologisée de maintes façons. Une première façon classique d'appréhender la question consiste à distinguer le mal naturel, qui renvoie aux cataclysmes justement «naturels » -

1. Pour un panorama des différentes interprétations possibles quant à la question de l'origine du mal, nous renvoyons au livre très éclairant de Barry Whitney (1989). 
cyclones, tremblements de terre, tsunamis (pour reprendre un exemple assez récent) - , qui produisent leur lot de victimes, du mal moral, qui renvoie plutôt aux vices humains. Mais une autre façon d'aborder le problème réside dans la distinction entre mal théorique et mal pratique ou, dit autrement, entre le mal pensé rationnellement et le mal vécu par des hommes, femmes et enfants. Cette distinction ouvre à la question de la pertinence existentielle de la théodicée. Celle-ci constitue-t-elle une entreprise qui aide les personnes victimes du mal à porter ou à surmonter ce dernier ou ne relève-t-elle pas plutôt d'une activité purement rationnelle et intellectuelle?

Dans le présent article, nous exposerons les grandes lignes du débat de la pertinence existentielle de la théodicée, sous l'angle des rapports entre théorie et pratique. Ce débat a été inauguré dans les années 1980 par deux auteurs travaillant aux États-Unis. Le premier, Kenneth Surin, a synthétisé sa pensée dans son œuvre maîtresse relative à ce sujet, Theology and the Problem of Evil (1986). Quant à la pensée du deuxième, Terrence W. Tilley, elle se retrouve dans son livre The Evils of Theodicy (1991). Ces deux auteurs critiquent avec véhémence la théodicée et son entreprise sur la base de leur manque de pertinence existentielle pour les personnes qui souffrent. Les thèses de Surin et de Tilley ont engendré des réactions sur la scène universitaire, réactions qui s'avèrent soit favorables, soit défavorables à la théodicée. Nous conclurons l'article par nos propres réflexions critiques sous l'angle des rapports entre théorie et pratique, réflexions qui, nous l'espérons, permettront de mieux clarifier certains des enjeux présents dans le débat.

\section{La pensée de Surin}

\subsection{La thèse}

La critique de Surin des théodicées s'appuie sur une distinction qu'il établit entre les théodicées d'approche théorique et les théodicées d'approche pratique. Si cet auteur critique avec véhémence les premières, il estime en revanche que les secondes offrent une pertinence sur le plan existentiel. Les théodicées théoriques se caractérisent par leur tentative d'articuler de manière cohérente et logique les affirmations de l'existence du mal, de l'existence de Dieu et du caractère infiniment bon et tout-puissant de ce dernier. Quant aux théodicées pratiques, leur mandat est davantage d'offrir des réponses qui soient parlantes pour les victimes du mal, en particulier en développant l'idée d'un Dieu salutaire qui agit de manière décisive pour contrer le mal (Surin 1986, 112). 


\subsection{Les théodicées théoriques}

Les théodicées théoriques s'inscrivent dans la foulée de l'entreprise classique de théodicée. Ce que Surin entend par entreprise classique de théodicée correspond à la manière usuelle de penser et de produire les théodicées depuis les Lumières. À l'époque, les philosophes et théologiens étaient préoccupés par une approche rationnelle en matière de théodicée, où l'objectif principal était de justifier Dieu du mal qui existe dans le monde. Or, les contemporains ont hérité de cette façon de problématiser cette question: «La théodicée, dans la forme classique ou canonique qu'on lui reconnaît d'ordinaire, est un exercice philosophique et/ou théologique comportant

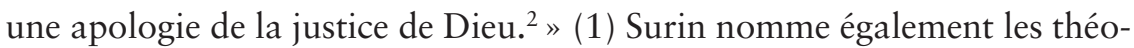
dicées qui découlent de cette perspective les «théodicées postleibniziennes". Parmi ce type de théodicées, il mentionne la Free Will Defence d'Alvin Plantinga, la Natural Law Theodicy de Richard Swinburne, la Soul-Making Theodicy de John Hick et la Process Theodicy d'Alfred North Whitehead. Nous n'entrerons pas dans le détail de chacune de ces théodicées ici.

L'idée clé de Surin pour illustrer la non-pertinence existentielle des théodicées théoriques s'exprime dans la phrase suivante: «Une théodicée postleibnizienne en est une qui conçoit la théodicée comme une entreprise essentiellement rationnelle ou théorique. Ainsi comprise, elle n'aurait aucune incidence sur quelque stratégie pratique destinée à vaincre le mal. » (13) Les théodicées théoriques se caractérisent donc par un souci de cohérence rationnelle ou logique et occultent la dimension pratique ou existentielle du mal.

\subsection{Les théodicées pratiques}

Les théodicées pratiques, quant à elles, ont pour trait commun le fait qu'elles font intervenir un Dieu de salut qui agit de façon décisive pour contrer le mal (112). Les pensées de Dorothee Soelle, de Jürgen Moltmann et de Peter Taylor Forsyth, que nous ne développerons pas ici, sont emblématiques de ce type d'approche. De façon générale, quatre idées maîtresses sous-tendent ces théodicées pratiques: 1) Dieu souffre avec ses créatures; 2) il ne peut être une divinité immuable et impassible; 3) la question centrale de la personne qui souffre est: «Dieu est-il un Dieu de salut, un Dieu qui peut m'aider? »; 4) une approche pratique en matière de théodicée,

2. La traduction des citations tirées d'ouvrages anglais est nôtre. 
qui appréhende le mal du point de vue des victimes, conduit à la sotériologie, plus précisément à une doctrine de la rédemption (137). C’est principalement en vertu du caractère sotériologique des théodicées pratiques que Surin estime que ces dernières ont plus de pertinence existentielle que les théodicées théoriques.

\subsection{Les arguments}

Surin relève plusieurs arguments pour mettre de l'avant le primat des théodicées pratiques sur les théodicées théoriques. Le premier concerne la conception de Dieu entretenue dans les théodicées théoriques. Étant donné qu'elles sont apparues à l'époque des Lumières, elles appréhenderaient Dieu de manière spécifiquement philosophique, dans un cadre théiste où l'accent est mis sur les attributs ontologiques divins, occultant ainsi des dimensions théologiques fondamentales au christianisme telle l'incarnation ${ }^{3}$. Un deuxième argument relève de l'ordre cosmique typique des Lumières. Les théodicées théoriques auraient hérité d'une façon d'appréhender la question du mal en insérant cette dernière dans l'ordre cosmique, le souci étant alors d'harmoniser "esthétiquement" la présence du mal avec l'univers. Une telle perspective néglige la dimension historique du mal et fait peu de cas qu'il existe des personnes dans l'histoire qui sont victimes du mal. Un troisième argument repose sur le fait que les théodicées théoriques n'auraient pas de dimension spécifiquement théologique, mais une logique uniquement. Pour Surin, la question du mal doit être absolument traitée dans un cadre théologique pour rejoindre l'expérience des personnes qui souffrent $^{4}$. Un quatrième argument avance que les théodicées théoriques sont anhistoriques, les théodicistes ${ }^{5}$ empruntant souvent leurs catégories conceptuelles à des auteurs classiques tels Augustin, Irénée ou Thomas d'Aquin. Lire ces auteurs à travers des préoccupations strictement métaphysiques ou philosophiques tel que le font les théodicistes théoriques constituerait donc une lecture qui s'avérerait infidèle à l'intention de ces auteurs classiques.

3. L'incarnation s'avère en effet fondamentale pour les théodicées pratiques étant donné qu'on y met de l'avant l'idée que Dieu souffre avec les victimes, dont l'événement de la Croix constitue une image paradigmatique.

4. Un cadre théologique permettra notamment de parler d'un Dieu qui donne sa grâce (104).

5. Par «théodicistes ", je réfère aux chercheurs ou aux penseurs qui élaborent ou ont élaboré des théodicées. Le terme anglais theodicists est couramment employé dans la littérature portant sur le sujet. 
Un cinquième argument tient au fait que les théodicées théoriques auraient une conception abstraite du mal, déconnectée de l'expérience des victimes. Un sixième argument est que l'entreprise théorique de théodicée comporte une certaine justification du mal: "Concevoir la théodicée comme un exercice purement théorique et scolaire revient à donner - fût-ce involontairement - une approbation tacite à la myriade de maux qui existent sur cette planète. » (50) Un septième argument avance que la théodicée théorique constitue une entreprise strictement individuelle et cognitive. Un huitième argument est que les théodicées théoriques tentent de saisir une réalité qui relève fondamentalement du mystère, en essayant de rendre intelligible ce qui ne l'est pas, c'est-à-dire le mal. Un neuvième argument relève le fait que les théodicées théoriques s'inscrivent dans une entreprise de logique uniquement, alors que, pour pouvoir avoir une pertinence existentielle, elles devraient avoir une dimension narrative.

Par ailleurs, deux arguments principaux sont invoqués par Surin pour démontrer le primat de l'approche pratique sur l'approche théorique en matière de théodicée. Le premier tient au fait que les théodicées pratiques mettent de l'avant l'idée d'une autojustification de Dieu sur la Croix. Pour Surin, l'automanifestation de Dieu sur la Croix justifie ce dernier du mal. Cette idée ramène à la conception traditionnelle selon laquelle, dans l'événement de la Croix, Dieu a souffert, et à ce titre, le mal ne peut lui être attribué. Qui plus est, la rédemption inaugurée par l'événement de la mort-résurrection de Jésus Christ constitue un événement de salut pour l'humanité: «La "réponse" chrétienne au "problème du mal" est la mise en discours, hésitante et balbutiante, de cette action réconciliatrice.» (16) Avec l'aspect salutaire vient la pertinence existentielle, et seules les théodicées pratiques revendiquent cet aspect salutaire, selon Surin. Une théologie de la Croix, qui engage un salut incarné par Dieu, s'avère donc capitale.

Le second argument d'importance concerne l'attention portée aux victimes dans les théodicées d'approche pratique. Ces théodicées pratiques accordent une plus grande attention à la réalité des victimes, puisqu'elles situent le mal du point de vue de ces dernières. Par exemple, contrairement aux théodicées théoriques qui, à la question du mal, offrent des réponses telles que: «le mal existe car les êtres humains sont libres et utilisent mal cette liberté ", les théodicées pratiques posent le problème d'une autre façon, notamment: «le mal existe car je suis présentement emprisonné, car je suis torturé, car j'ai faim, etc. » (145). Les théodicées pratiques ont donc, à ce titre, une pertinence existentielle plus grande que les théodicées théoriques. 


\subsection{La limite de tout type de théodicée}

Malgré la prééminence des théodicées pratiques sur les théodicées théoriques, Surin estime que même les premières se butent à des limites fondamentales. Ces limites viennent du fait que face aux victimes du mal le plus tragique, aucune réponse ne peut s'avérer satisfaisante. Le cas d'Auschwitz en est un bon exemple. Pour Surin, même un discours sur la rédemption se trouve incapable de répondre à la question du mal telle qu'elle s'est posée par rapport à Auschwitz. Relatant un triste épisode où des enfants ont été jetés vivants dans un four crématoire, Surin affirme que: "aucune affirmation, théologique ou autre, ne devrait être formulée qui ne serait pas crédible en présence des enfants jetés au feu» (17). Ainsi, pour cet auteur, la primauté des théodicées pratiques sur les théodicées théoriques ne signifie donc pas que les premières constituent des solutions parfaites au problème du mal.

\section{La pensée de Tilley}

\subsection{La thèse}

Notre deuxième auteur principal est Terrence W. Tilley. L'essentiel de sa pensée se trouve dans le livre The Evils of Theodicy (1991). Tout comme Surin, Tilley critique vivement l'entreprise traditionnelle de théodicée. En fait, il pousse encore plus loin sa critique dans la mesure où il estime que cette entreprise devrait être complètement abandonnée. Selon lui, la théodicée est non seulement incapable de répondre à la question du mal, mais elle crée des «maux nouveaux»: « ... la théodicée, en tant que pratique de discours, doit être abandonnée, car la pratique de la théodicée n'épargne pas du mal, mais en occasionne » $(1991,5)$. Tilley avance que la théodicée est une entreprise intellectuelle qui occulte un type de mal, à savoir le mal social, et pour cet auteur, l'occultation d'un tel mal constitue un mal en soi.

\subsection{L'argumentation}

L'ouvrage de Tilley est divisé en trois parties principales. Dans le cadre du présent article, nous allons nous attarder principalement sur la troisième, puisque c'est là que se trouve le cœur de sa pensée. La première partie expose la théorie du langage de John R. Searle, qui ne sera pas développée ici. Retenons qu'en présentant cette théorie, Tilley veut montrer que la théodicée, comme discours ou langage, a un impact « réel » sur les croyants: 
La théorie des actes du langage met en lumière les actions que nous accomplissons en parlant et propose une manière de comprendre leur pouvoir et leur valeur morale. Elle offre une grille qui permet de montrer comment nous employons les mots pour affirmer, créer, diminuer, obscurcir et résoudre les problèmes liés au péché et à la souffrance. (22)

Quant à la deuxième partie, elle constitue une application de cette théorie du langage à des textes classiques en matière de théodicée, notamment le livre biblique de Job, l'Enchiridion d'Augustin et les Dialogues Concerning Natural Religion de Hume. Dans cette partie, Tilley veut démontrer qu'ayant été lus dans une perspective de théodicée, ces textes ont été "mal lus ", puisque l'intention première de leurs auteurs n'était pas de développer des théodicées à proprement parler, donc de justifier Dieu de l'existence du mal. Or, en étant lus dans une optique de théodicée, la voix de ces textes aurait été "étouffée ». L'entreprise de théodicée aurait ainsi pour conséquence de bâillonner d'autres voix, ce qui en fait une entreprise néfaste et qui doit être abandonnée.

Les deux premières parties du livre de Tilley ne font en quelque sorte qu'introduire à la troisième, beaucoup plus fondamentale, sur le plan de l'argumentation, pour bien saisir l'essentiel de la pensée de cet auteur.

\subsubsection{Trois facteurs issus des Lumières}

Tilley entame cette troisième partie en parlant de l'origine historique de la théodicée. Tout comme Surin, il situe cette origine à l'époque des Lumières. Trois facteurs émanant de cette période auraient facilité l'émergence de l'entreprise de théodicée. D’abord, les Lumières ayant été notamment marquées par une contestation des autorités religieuses, un premier facteur se retrouve dans la rationalisation des doctrines religieuses, les Églises ayant dû réagir à l'attaque de la modernité. Un deuxième facteur concerne l'importance du théisme providentiel philosophique dans les débats religieux des Lumières. Dieu prend donc un visage davantage philosophique que théologique. Quant au troisième facteur, il s'agit des bouleversements cosmologiques amenés par la révolution scientifique inaugurée par Galilée et Newton. À l'intérieur d'un tel cadre scientifique, la question à se poser quant au mal est de savoir comment celui-ci peut s'insérer dans un univers régi par des lois mécaniques parfaites. Ces trois facteurs ont permis l'émergence de l'entreprise de théodicée, entreprise qui envisage la question du mal de manière théorique, abstraite et non spécifiquement théologique (224). 


\subsubsection{Un discours «impratique»}

Pour Tilley, la théodicée serait un discours «impratique " (impractical practice). La raison à cela tiendrait au fait qu'elle constitue une entreprise essentiellement théorique:

La théodicée est une pratique de discours qui est «impratique ». En d'autres termes, elle est une pratique purement théorique répondant à des problèmes théoriques, et non une théorie pratique répondant à de problèmes concrets qui se posent dans la pratique religieuse. Les théodicées ne répondent pas aux plaintes et aux lamentations. Elles ne sont pas adressées aux gens qui pèchent et souffrent. Elles sont adressées à des intellects individuels abstraits qui ont des problèmes purement théoriques à comprendre le mal. (229)

Le fait que la théodicée s'attache à répondre à une interrogation foncièrement théorique ferait du théodiciste un être détaché qui problématise la question du mal sans référence à un cadre existentiel ou théologique. Tilley condamne également une telle attitude. Pour lui, la théodicée constitue un problème d'abord et avant tout pratique: "Même si les théodicées donnaient des réponses légitimes aux problèmes théoriques, le vrai problème n'est pas théorique. » (232) Le caractère théorique, ou «impratique ", de la théodicée est un autre argument pour appuyer la non-pertinence existentielle de la théodicée, selon Tilley.

\subsubsection{La théodicée comme déclaration assertive}

Une des caractéristiques majeures de la théodicée, en lien avec son aspect théorique, serait qu'elle fait du mal une abstraction (234). De la sorte, elle détourne le regard des multiples situations où les humains sont des victimes concrètes du mal: «les théodicées obscurcissent "la singularité radicale et impitoyable du mal humain" et constituent une "pratique" qui détourne ses regards des cruautés qui existent dans ce monde " (ibid.). Or, en ne situant pas le mal "au bon endroit", la théodicée créerait ainsi du mal. C'est en vertu du fait que la théodicée relèverait du type de langage de la déclaration assertive qu'elle recèle un tel potentiel pour produire du mal. La théodicée est assertive, puisqu'elle dit ce qu'est le mal, et déclarative, puisqu'en affirmant ce qu'est le mal, elle amène un changement dans le monde, à savoir celui d'occulter certains types de mal et ainsi de créer d'autres types de mal. Tilley résume son argumentation de la manière suivante:

En résumé, la théodicée est un acte du langage dans lequel les théodicistes ne décrivent pas, mais déclarent ce qui est mal. Cependant, s’ils ne déclarent pas 
mal ce qui l'est réellement, leurs déclarations créent une réalité dans laquelle ce qui est réellement mal ne l'est pas. Si les théodicistes se trompent dans ce qu'ils déclarent mal, ils créent un discours inconsistant et, en dernière analyse, destructeur [...] [É]crire une théodicée, c'est faire [perform] une déclaration assertive qui est en fait une déclaration falsifiante qui occulte les maux véritables. (235)

Ainsi, pour Tilley, non seulement la théodicée manque de pertinence existentielle, mais elle crée du mal.

\subsubsection{L'occultation du mal social}

Le mal principal qu'occulterait la théodicée est le mal social:

En général, une société, une pratique sociale ou une situation sociale peut être reconnue comme oppressante si elle est structurée de façon à ce que de bonnes personnes accomplissent ordinairement des actes qu'elles croient bons, mais qui ordinairement et involontairement en blessent d'autres. Une société ou une situation sociale est injuste si elle est structurée de façon à ce que de bonnes personnes accomplissement ordinairement des actes qu'elles croient bons, mais qui involontairement privent d'autres de ce qui leur est dû [...]. Le point à retenir est le suivant: s'engager dans la pratique de la théodicée implique que l'on lise des textes - voire des textes qui traitent du mal et qui tentent de miner la théodicée - de façon à mettre en valeur certains maux comme importants et d'occulter d'autres formes de mal [...]. Les théodicistes ne reconnaissent pas que certaines pratiques ou structures causent du mal au moyen de bonnes actions. (238)

La raison pourquoi le mal social est pratiquement invisible aux yeux des théodicistes proviendrait du fait que les théodicées ont hérité d'une typologie selon laquelle le mal est de deux types: moral (ou humain), ou physique (ou naturel). Dans le premier type, la cause du mal est attribuée aux humains (ex.: vices), alors que, dans le deuxième, elle est attribuée à la nature (ex.: cataclysmes naturels). Le mal social, complexe et subtil, ne figure donc pas dans cette typologie. D'où l'idée avancée par Tilley que, pour les théodicistes, le mal social n'existe pas. L'entreprise de théodicée nous rend ainsi aveugles au mal social: «La théodicée nous rend littéralement aveugle face aux maux sociaux de notre époque. » (246) La théodicée constitue donc aux yeux de Tilley une pratique de discours qui procède d'une misdeclaration of evil (247). Et cette dernière s'avère un mal majeur: "Si les théodicistes se trompent dans ce qu'ils déclarent mal, ils créent un discours destructeur» (248). 


\subsubsection{La solution envisagée: un acte de relecture}

Pour enrayer l'impact négatif des théodicées, Tilley suggère de relire les textes qui ont traditionnellement été lus dans une perspective de théodicée et de les interpréter autrement, c'est-à-dire de manière non déclarative, ou du moins de situer adéquatement ce que ces textes veulent réellement déclarer: "nous pouvons employer des textes pour interrompre le processus déclaratif de la théodicée, pour révéler les véritables dimensions du monde qu'il déclare, pour reconstruire les traditions qu'il efface et pour contrer ses forces. » (249) Et une telle relecture, réinterprétation et réutilisation des textes doit se situer dans une perspective clairement plus pratique que théorique, faire ressortir la complexité du mal et, surtout, ne point tenter de rendre invisibles certains types de mal: «Nos propres actes symboliques ne doivent pas occulter les maux, mais les identifier dans leurs multiples formes, comprendre les processus qui les produisent, repérer les discours qui les révèlent et donner pleins pouvoirs aux praxis de réconciliation qui le vaincront. » (34) Ainsi, selon Tilley, l'entreprise de théodicée doit absolument être contrecarrée, puisqu'elle constitue un projet qui n'offre aucun soutien aux personnes qui souffrent et génère du mal.

\section{Les réactions}

Surin et Tilley estiment tous deux que les théodicées s'avèrent non pertinentes existentiellement. Pour le premier, cette impertinence existentielle concerne les théodicées théoriques, alors que pour le deuxième, elle se traduit dans le caractère néfaste des théodicées. Leurs thèses ont entraîné un certain nombre de réactions dans l'arène académique. Nous allons nous attarder maintenant aux réactions qui ont trait aux rapports entre théorie et pratique, ces derniers étant fondamentaux pour le débat.

\subsection{David O’Connor}

David O’Connor critique avec véhémence la position de Surin. Il expose dans son article «In Defense of Theoretical Theodicy (1988) l'essentiel de sa pensée sur le sujet. Pour cet auteur, les critiques de Surin à l'endroit de la théodicée sont insuffisantes, alors que lui considère cette dernière comme une entreprise philosophique et théologique pertinente.

O'Connor estime qu'il existe en fait deux problèmes à la question du mal, à savoir un problème théorique ou abstrait, et un problème pratique ou existentiel. Vu ces deux types de problèmes, il faut concevoir deux types de 
réponses, l'un relevant de la logique et l'autre de la pragmatique $(1988,63)$. Tout comme Surin, O’Connor est d'accord avec l'idée que la théodicée constitue un discours d'abord et avant tout théorique. Cela dit, ce qu'il constate chez Surin est que ce dernier perçoit les théodicées théoriques comme devant répondre au problème pratique ou existentiel du mal. O'Connor estime qu'il y a là une erreur de fond, la théodicée ne visant pas à donner une réponse existentielle aux personnes qui souffrent, mais à offrir une explication logique au problème du mal.

Même s'il distingue le volet théorique du volet pratique, O'Connor estime tout de même que les deux se complètent, que l'un ne va pas sans l'autre. Selon lui, la religion contient ces deux pans, chacun ayant son utilité. Son volet théorique, qui concerne l'aspect systématique de la foi, a un impact sur son volet pratique ou existentiel, ce dernier ayant trait à l'aspect vécu de la foi. La foi vécue trouve son sens dans l'horizon d'un système religieux régi par une structure et une cohérence. C’est pourquoi les théodicées (théoriques) ont leur pertinence. Celle-ci n'est certes pas d'ordre existentiel, puisque tel n'est pas le mandat de ces productions de toute façon.

\subsection{James Wetzel}

Dans son article «Can Theodicy Be Avoided? The Claim of Unredeemed Evil» (1989), James Wetzel soutient son idée maîtresse selon laquelle ni une théodicée théorique ni une théodicée pratique ne peuvent répondre adéquatement et complètement à la question du mal. S’il est vrai que la théodicée théorique n'affiche pas de pertinence existentielle, la théodicée pratique également accuse un retard en ce sens. Wetzel critique donc la thèse de Surin. Se référant à la théodicée "pratique » de Dorothee Soelle, qui recommande de partager les souffrances des victimes, il souligne que même si une telle attitude est louable, elle ne parvient pas plus à répondre à la question du mal, qui est le mandat fondamental de l'entreprise de théodicée $(1989,12)$. La solidarité avec les victimes laisse donc la question du mal ouverte. Étant donné que ni le volet théorique ni le volet pratique ne permettent d'appréhender la question du mal dans sa globalité, les deux sont nécessaires, selon lui.

\subsection{Michael J. Quirk}

Dans une recension qu'il a faite du livre de Surin, Michael J. Quirk affirme que le dénigrement de l'aspect théorique des théodicées chez le premier s'avère 
non fondé. Même l'optique d'une théologie de la Croix, que revendique Surin pour surmonter le problème du mal, se base sur un élément théorique (1987, 2). Ainsi, selon lui, la théorie constitue elle-même une forme de praxis:

Les théories philosophiques au sujet du monde sont aussi historiques que n'importe quelle théorie et, tout comme d'autres modes de praxis, elles se transforment rationnellement sur fond d'un changement de contexte ou d'un besoin commun. Les théories - les théories philosophiques - sont une forme de praxis, et aussi longtemps qu'elles peuvent rationnellement interpréter, articuler et justifier provisoirement les demandes de la foi, elles sont un mode de praxis de loin plus indispensable que, je pense, Surin le conçoit. (ibid.)

\subsection{Michael Scott}

Dans son article intitulé "The Morality of Theodicies " (1996), Michael Scott réagit notamment à O'Connor. Selon lui, il n'est pas toujours possible de catégoriser les situations comme soit d'ordre théorique, soit d'ordre pratique, catégorisation que fait O'Connor. D'après Scott, il existe plusieurs cas « intermédiaires ", qui ne sont ni uniquement théoriques ni uniquement pratiques: "Il semble plausible de postuler que plusieurs exemples de "problèmes" du mal partageront des éléments à la fois des problèmes théoriques classiques et des problèmes pratiques du mal. » $(1996,4)$ Scott donne notamment l'exemple d'une personne qui voit dans un bulletin de nouvelles télévisé l'annonce des morts occasionnées par un désastre naturel et qui en vient à rejeter l'existence de Dieu sur cette base. Pour cette personne, qui n'est que témoin du drame, un tel rejet de l'existence de Dieu peut constituer soit une réponse existentielle, soit une réponse rationnelle.

\subsection{Todd Billings}

La position de Todd Billings se situe à la suite des travaux de Surin et de Tilley, et se retrouve dans ses articles "Theodicy as a "Lived Question:" Moving Beyond a Theoretical Approach to Theodicy " (2000a) et "After Tilley: The Sustaining Relevance of the Problem of Suffering as a Concrete Question » (2000b). Même si, de manière générale, il appuie les thèses de ces auteurs, il estime néanmoins que la théodicée occupe une place centrale pour la pratique chrétienne, en tant que réflexion sur le mal. Le cœur de sa pensée réside dans l'affirmation que la question du mal doit rester ouverte.

Même si la théodicée peut d'abord et avant tout sembler relever du domaine théorique, Billings considère qu'elle concerne en fait surtout le 
champ pratique, vu son importance pour les croyants qui souffrent. L'auteur cite ici Moltmann: «Plus une personne croit, plus profondément elle expérimente de la douleur devant la souffrance qui existe dans le monde, et plus passionnément elle s'interroge sur Dieu et la nouvelle création $^{6}$. ${ }^{2}$ La théodicée constituerait donc essentiellement une interrogation pratique.

En raison de son allégeance pratique, Billings soutient que la théodicée devrait être une question "vécue ». Poser la question de la théodicée constitue une manière de vivre dans le monde en tenant compte de l'existence du mal (2000a, 3). S'engager activement pour atténuer la souffrance des victimes est une façon pour le chrétien de poser la question de la théodicée. Or, comme il a été mentionné plus haut, la réponse à cette question doit demeurer ouverte en tout temps, car seule une réponse ouverte peut inciter à l'engagement actif auprès des personnes qui souffrent. Billings croit en effet que de donner des réponses «fermées" ou absolues à la question du mal ne peut conduire bien souvent qu'à l'inaction et à la résignation:

... les personnes qui refusent d'agir en réponse à la souffrance - comme ceux qui ignorent les sans-abris - pratiquent en fait une théodicée. Pour ces personnes, la souffrance de l'autre n'est pas une question brute et ouverte - elle peut être clairement expliquée par des arguments sociologiques et psychologiques [...]. La question brute et ouverte de la souffrance est une dimension manquante de la pratique de tous ceux qui expliquent et oublient la souffrance de l'autre. $(2000 \mathrm{a}, 4)$

\section{Réflexions critiques sous l'angle des rapports entre théorie et pratique}

Il n'est pas facile de se positionner dans un tel débat, puisque chaque auteur amène des éléments qui ont leur pertinence. La question des rapports entre théorie et pratique est elle-même l'objet de maintes études et réflexions chez les chercheurs et penseurs en sciences humaines et sociales. Toutefois, nous allons tenter d'établir notre point de vue en commentant chacune des positions qui ont été exposées ici.

Surin distingue d'emblée l'approche théorique de l'approche pratique en matière de théodicée. Pour schématiser sa pensée, les théodicées théoriques n'affichent aucune pertinence existentielle, étant strictement d'ordre rationnel et cognitif, alors que les théodicées pratiques ont quant à elles une pertinence existentielle. Toutefois, nous mettons en question la typologie de

6. Billings 2000a, 1, qui cite Moltmann 1993, 49. 
Surin. Si nous reconnaissons que ce qu'il nomme "théodicées théoriques» correspond effectivement à des théodicées, nous en sommes bien moins certains en ce qui a trait aux théodicées pratiques. Soelle et Moltmann peuvent-ils réellement être considérés comme des théodicistes et leur pensée respective sur le mal comme des théodicées? Si Surin définit la théodicée comme une entreprise de justification de Dieu du mal qui existe dans le monde, nous voyons mal en quoi une pensée qui recourt à une théologie de la Croix peut être appréhendée comme théodicée, ce type de théologie relevant davantage de la sotériologie.

De plus, même si dans la littérature relative à ce débat la plupart des auteurs impliqués traitent des positions de Surin et de Tilley comme s'inscrivant dans une même lignée — ce qui n'est pas faux de manière générale nous estimons qu'il existe une différence notable entre les deux qui n'est pas prise en compte. Surin, pour sa part, opère une distinction radicale entre la théorie et la pratique. Quant à Tilley, il estime que la théorie engendre un certain type de pratique. Pour lui, la théodicée classique n'est pas que théorique, mais elle est également pratique puisqu'elle constitue une pratique néfaste qui produit du mal. Les implications d'une telle vision quant aux rapports entre théorie et pratique pourraient expliquer pourquoi Tilley est plus radical dans sa condamnation des théodicées que ne l'est Surin.

En ce qui concerne la position d'O'Connor, qui répond aux attaques faites à la théodicée quant à sa non-pertinence existentielle, il soutient que de telles attaques sont non fondées puisque le mandat de la théodicée n'est pas de répondre à une interrogation pratique ou existentielle, mais théorique. Une telle position présuppose une distinction des domaines entre ce qui relève du champ théorique et ce qui relève du champ pratique. Mais là encore, la pensée d'O'Connor pose question. Qu'un discours vise principalement à répondre à un questionnement théorique le justifie-t-il totalement de toute responsabilité pratique ou morale?

La pensée de Wetzel nous semble quant à elle fort intéressante. Celuici estime que les théodicées théoriques et pratiques sont toutes deux nécessaires, chacune permettant de développer un aspect différent de la question. De plus, les limites qu'il attribue à chacune nous apparaissent judicieuses: les théodicées théoriques ne peuvent répondre à la question du mal injustifiable, alors que les théodicées pratiques, même si elles peuvent lui donner un sens, ne peuvent offrir d'explication à la souffrance des personnes. En effet, prôner une solidarité envers les victimes ne répond en rien à la question du pourquoi de l'existence du mal. 
Nous trouvons également pertinent le commentaire de Quirk. La plupart des détracteurs de la théodicée reprochent à cette dernière d'être trop théorique et intellectualiste. Or, comme le souligne si bien Quirk, même l'optique d'une théologie de la Croix préconisée par les tenants de la théodicée pratique est fondamentalement théorique. En effet, de dire que la souffrance de Dieu sur la croix justifie ce dernier de l'existence du mal dans le monde repose non d'abord et avant tout sur des considérations pratiques, mais sur des considérations théoriques et idéelles!

L'apport de Scott nous paraît également important. Comme lui, nous considérons qu'il n'est pas toujours évident de déterminer ce qui relève de l'ordre théorique et ce qui relève de l'ordre pratique. Il n'est pas toujours possible de diviser la réalité selon le schème théorie-pratique. Et même dans un tel cadre, de multiples combinatoires sont possibles entre ces deux ordres.

Nous estimons aussi très pertinente la perspective de Billings. Aborder les aspects théoriques et pratiques de la théodicée de manière à rendre la question "vécue » et ouverte nous semble entrer en conformité avec l'expérience réelle. Car le mal constitue non seulement un concept abstrait, mais il est expérimenté par les êtres humains de manière entière, que ce soit sur les plans physique, émotionnel, affectif, psychique, intellectuel ou cognitif. Qui plus est, la position de Billings appelle à une humilité qui nous semble essentielle. Malgré toutes nos tentatives humaines pour comprendre le pourquoi du mal, force est d'admettre que nous ne parviendrons sans doute jamais à répondre à cette question totalement.

\section{5. À l'horizon du débat: une vision binaire}

Il est possible de voir dans nos commentaires que nous sommes favorable à une manière d'appréhender la question du mal qui ne scinde pas radicalement ses dimensions théorique et pratique. Selon nous, le binôme théoriepratique constitue une façon d'aborder le problème, un angle de vue, une façon de lire la réalité, qui ne doit pas être confondue avec la réalité ellemême cependant. Nous estimons que le monde n'est ni uniquement pratique ni uniquement théorique, mais il est les deux à la fois, d'où l'importance de toujours chercher à articuler ensemble ce couple, même s'il n'est pas toujours possible de déterminer ce qui relève de chacune de ces dimensions. Pour ce qui nous incombe présentement, nous critiquons donc la vision radicale de Surin selon laquelle les théodicées théoriques sont non pertinentes existentiellement et les théodicées pratiques, pertinentes existentiellement. 
Nous critiquons tout autant cette de Tilley qui ne voit dans la théodicée qu'une entreprise fallacieuse de production du mal.

De manière générale, le débat de la pertinence existentielle de la théodicée semble reposer sur des arguments binaires, ce qui nous paraît malheureux. Le présent article n'a traité que d'un aspect de ce débat, à savoir la question des rapports théorie-pratique. Mais pour ce qui est de l'ensemble du débat, cette bipolarité se caractérise de manière générale par les affirmations schématiques suivantes: la théodicée est soit théorique, soit pratique; elle est soit théologique, soit philosophique; elle est soit morale, soit immorale; et enfin, elle est soit pertinente existentiellement, soit non pertinente existentiellement. Nous critiquons bien sûr une telle façon d'aborder le problème, qui ne rend pas compte de la complexité de la question du mal, voire de la complexité du réel.

\section{Conclusion}

En conclusion, nous aimerions partager une réflexion sur ce débat de la pertinence existentielle de la théodicée. Si ce débat a suscité des réactions si vives ainsi que des positions si polarisées, cela est peut-être dû au fait que la problématique de la théodicée touche fondamentalement à la question du sens que les êtres humains donnent ou essaient de donner à leur vie. En effet, selon nous, l'entreprise de théodicée s'inscrit dans cette quête de sens. La question à laquelle elle tente de donner une réponse, à savoir pourquoi le mal existe si Dieu est infiniment bon et tout-puissant, s'enracine dans la dynamique d'une existence humaine qui cherche à comprendre et à se comprendre. Au-delà de la querelle universitaire opposant ceux qui estiment que la théodicée constitue une entreprise appréciable et utile et ceux qui croient le contraire, force est d'admettre que chacun des camps essaie d'apporter un élément de réponse éclairant ou cernant un aspect spécifique de la question du mal, et que ni l'un ni l'autre ne parviendra vraisemblablement à la résoudre de manière totalement satisfaisante. Pourquoi le mal si Dieu est infiniment bon et tout-puissant ? Là se trouve peut-être la question fondamentale à la source de la théologie chrétienne. 


\section{Références}

Billings, T. (2000a), "Theodicy as a "Lived Question:" Moving Beyond a Theoretical Approach to Theodicy ", Journal for Christian Theological Research, 5/2, accessible en ligne à l'adresse <www.luthersem.edu/ctrf/ JCTR/Vol05/billings.htm> (consultée le 18 juin 2007).

(2000b), «After Tilley : The Sustaining Relevance of the Problem of Suffering as a Concrete Question »/ texte d'une communication donnée lors de l'assemblée annuelle du Christian Theological Research Fellowship, Nashville, 2000, accessible en ligne à l'adresse <www.luthersem.edu/ctrf/Papers/2000_Billings.htm> (consultée le 18 juin 2007).

Moltmann, J. (1993), The Trinity and the Kingdom, Minneapolis, Fortress Press.

O'Connor, D. (1988), «In Defense of Theoretical Theodicy», Modern Theology, 5/1, p. 61-74.

Quirk, M.J. (1987), «Theology and the Problem of Evil, by Kenneth Surin » (recension), Theology Today, 44/3, accessible en ligne à l'adresse <http://theologytoday.ptsem.edu/oct1987/v44-3-bookreview13.htm> (consultée le 18 juin 2007).

Scott, M. (1996), "The Morality of Theodicies ", Religious Studies, 32, p. 1-13.

SuRIN, K. (1986), Theology and the Problem of Evil, Oxford / New York, Blackwell.

Tilley, T.W. (1991), The Evils of Theodicy, Washington, Georgetown University Press.

Wetzel, J. (1989), "Can Theodicy be Avoided? The Claim of Unredeemed Evil », Religious Studies, 25, p. 1-13.

Whitney, B.L. (1989), What Are They Saying about God and Evil?, Mahwah, Paulist Press. 


\section{Résumé}

Dans les années 1980, deux auteurs, Kenneth Surin et Terrence W. Tilley, ont amorcé un débat quant à la pertinence existentielle de la théodicée, en critiquant fortement l'entreprise de cette dernière. Leurs positions ont provoqué de vives réactions dans l'arène universitaire, celles-ci s'avérant soit favorables, soit défavorables à la théodicée. L'article expose les positions des principaux protagonistes du débat et propose quelques réflexions sur le thème des rapports entre théorie et pratique qui s'y dégage. À l'horizon de ce débat se dessine une vision binaire des choses, que l'auteur critique pour dénouer les impasses actuelles dans lesquelles celui-ci semble être plongé.

\section{Abstract}

In the 1980s, scholars Kenneth Surin and Terrence W. Tilley inaugurated the debate of the existential relevance of theodicy through a firm criticism of the latter enterprise. Their positions triggered a number of reactions, both favourable and unfavourable toward theodicy, on the academic scene. This article presents the main positions of that debate and reflects on the relationships between theory and practise that stem out of it. Underlying this debate lies a binary vision of things which the author criticizes in order to surmount the pitfalls in which the debate seems to be put. 